\title{
$\operatorname{CONF}-9605 / 01--3$
}

UCRL-JC-122657

PREPRINT

\section{Laser Isotope Purification of Lead for Use in Semiconductor Chip Interconnects}

Karl Scheibner, Chris Haynam, Earl Worden, Brad Esser

RECEIVED

MAR 181996

OSTI

This paper was prepared for submittal to the 46th Electronic Components and Technology Conference

Orlando, Florida

May 28-31, 1996

This is a preprint of a paper intended forpublication in a journal or proceedings. Since changes may be made before publication, this preprint is made available with the understanding that it will not be cited or reproduced without the permission of the author.

\section{. $\quad$ February 1996}

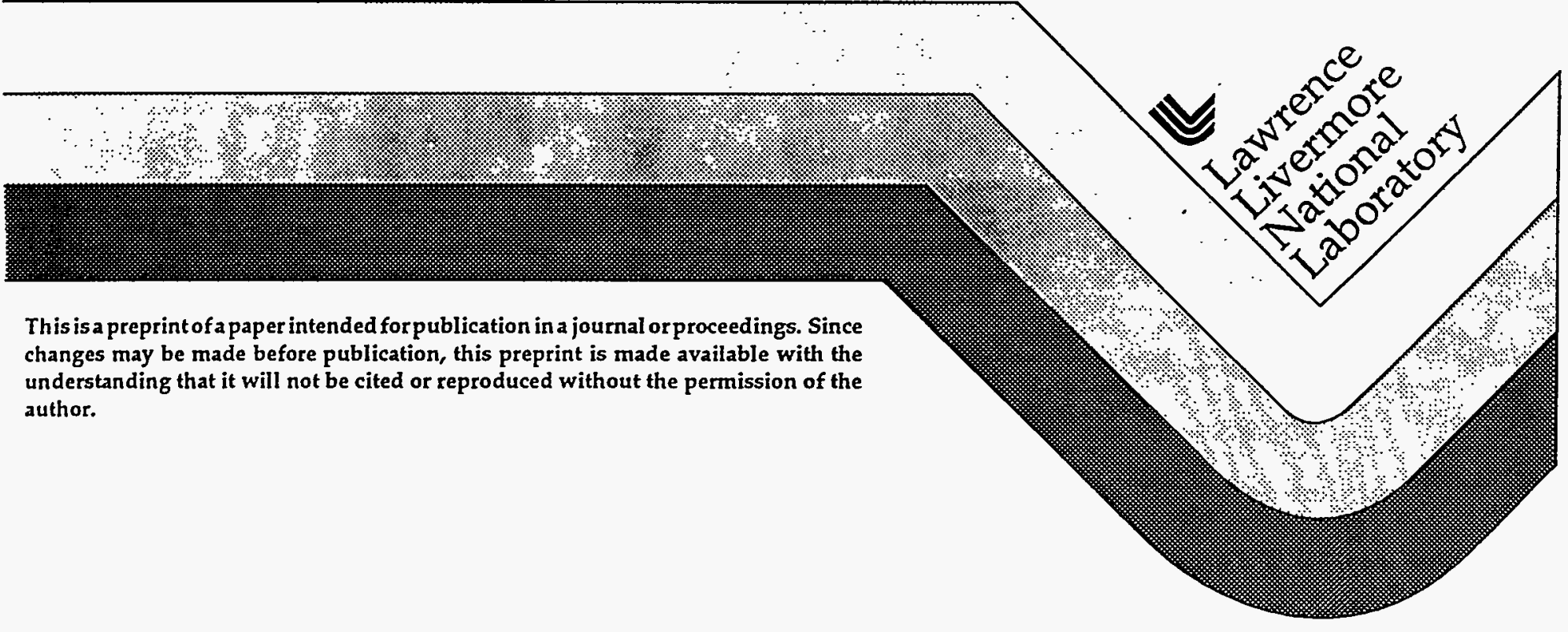




\section{DISCLAIMER}

This document was prepared as an account of work sponsored by an agency of the United States Government. Neither the United States Government nor the University of California nor any of their employees, makes any warranty, express or implied, or assumes any legal liability or responsibility for the accuracy, completeness, or usefulness of any information, apparatus, product, or process disclosed, or represents that its use would not infringe privately owned rights. Reference herein to any specific commercial product, process, or service by trade name, trademark, manufacturer, or otherwise, does not necessarily constitute or imply its endorsement, recommendation, or favoring by the United States Government or the University of California. The views and opinions of authors expressed herein do not necessarily state or reflect those of the United States Government or the University of California, and shall not be used for advertising or product endorsement purposes. ' 


\title{
Laser Isotope Purification of Lead for Use in Semiconductor Chip Interconnects
}

\author{
Karl Scheibner, Chris Haynam, Earl Worden, and Brad Esser \\ Lawrence Livermore National Laboratory \\ Livermore, CA, USA \\ email: scheibner@llnl.gov
}

\begin{abstract}
Lead, used throughout the electronics industries, typically contains small amounts of radioactive ${ }^{210} \mathrm{~Pb}$ (a daughter product of the planet's ubiquitous ${ }^{238} \mathrm{U}$ ) whose ${ }^{210}$ Po daughter emits an $\alpha$-particle that is known to cause soft errors in electronic circuits. The ${ }^{210} \mathrm{~Pb}$ is not easily separable by chemical means. This paper describes the generic Atomic Vapor Laser Isotope Separation (AVLIS) process developed at the Lawrence Livermore National Laboratory (LLNL) over the last 20 years, with particular emphasis on recent efforts to develop the process physics and component technologies required to remove the offending ${ }^{210} \mathrm{~Pb}$ using lasers. We have constructed a developmental facility that includes a process laser development area and a test bed for the vaporizer and ion and product collectors. We will be testing much of the equipment and demonstrating pilot-scale AVLIS on a surrogate material later this year. Detection of the very low alpha emission even from commercially available low-alpha lead is challenging. LLNL's detection capabilities will be described. The goal of the development of lead purification technology is to demonstrate the capability in FY97, and to begin filling orders of up to several MT/y of isotopically purified material in FY98.
\end{abstract}

\section{Introduction}

Lead is a key material used in the Controlled Collapsed Chip Connection (C4) technique that is used to form connections between the IC chip and the package. One isotope of lead, ${ }^{210} \mathrm{~Pb}$, is radioactive with a half-life of $22.3 \mathrm{y}$, formed as the by-product of uranium decay, and is found in low concentrations in lead ore. The alpha emissions that come from the ${ }^{210} \mathrm{Po}$ daughter of ${ }^{210} \mathrm{~Pb}\left({ }^{210} \mathrm{~Pb} \rightarrow{ }^{210} \mathrm{Bi}+\beta \rightarrow\right.$ $\left.{ }^{210} \mathrm{Po}+\beta \rightarrow{ }^{206} \mathrm{~Pb}+\alpha\right)$ can cause soft errors at unacceptable rates in the memory or logic circuitry of the integrated chip. This may place limitations on $\mathrm{C} 4$ usefulness or impose design constraints in future generations of ICs, particularly as reductions in feature size and operating voltage increase sensitivity to alpha emissions.

The AVLIS Program at LLNL is developing a capability of isotopically purifying lead for these applications by applying our uranium isotope separation technology.

\section{History of he AVLIS Program at LLNL}

Over the past 20 years AVLIS has developed a laser-based method for separating isotopes. As shown schematically in Fig. 1, an element is heated within a vacuum vessel with either an electron beam or, in the case of more volatile materials, resistance heater. Material vaporizes and streams upward forming a low density atomic vapor. This vapor is illuminated

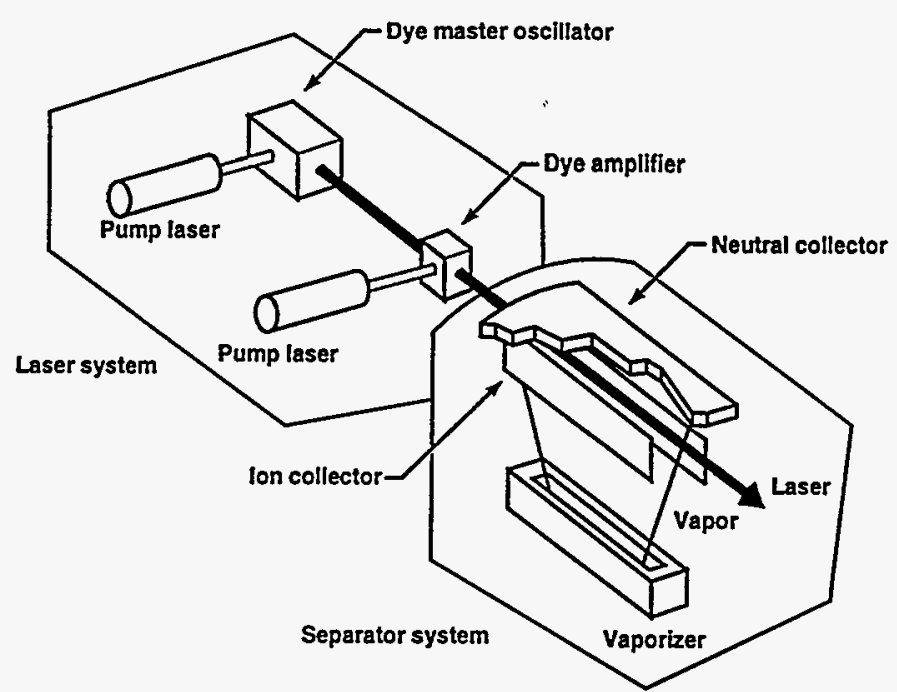

with multiple laser wavelengths and is step-wise resonantly excited until it has enough energy to ionize. The lasers are set at precisely controlled frequencies and have sufficiently narrow frequency bandwidth to ionize a single isotope, in this case ${ }^{210} \mathrm{~Pb}$. The resulting isotopically-enriched plasma, formed between pairs of charged extractors, is electrostatically separated and condensed. This "waste" material is enriched in the isotope selected by the laser and is typically called the Product. The remaining "depleted" neutral vapor stream, called the Tails stream, is condensed on the Tails Collector. This general method of separation a Feed stream into an enriched Product stream and a depleted Tails stream is called AVLIS.

The focus of the AVLIS program has been the enrichment of uranium for use in commercial nuclear reactors. A cut-away of our enrichment facility is shown in Fig. 2. Plant scale

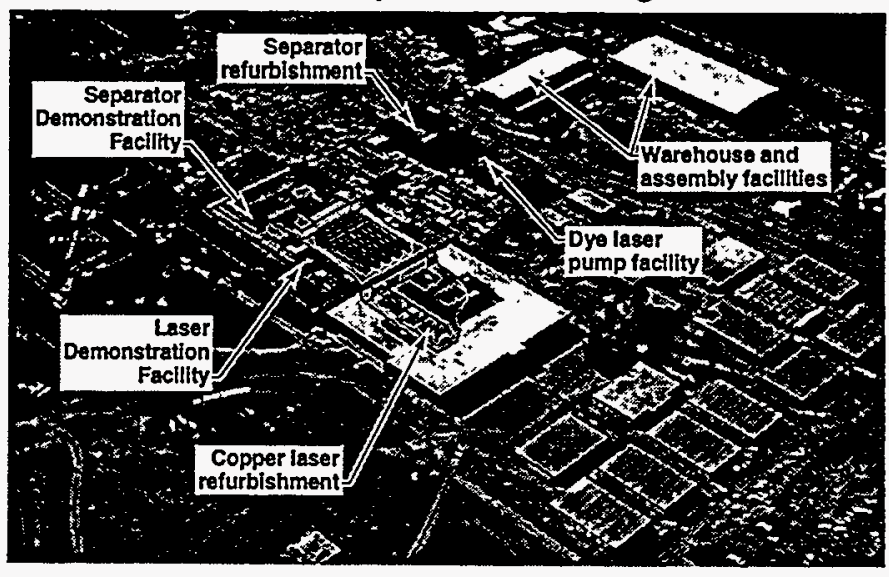

component technologies have been demonstrated, including copper lasers and refurbishment, dye lasers, separators and refurbishment, and integrated system controls. Shown in Fig. 3 
are our copper lasers, which convert electrical energy to

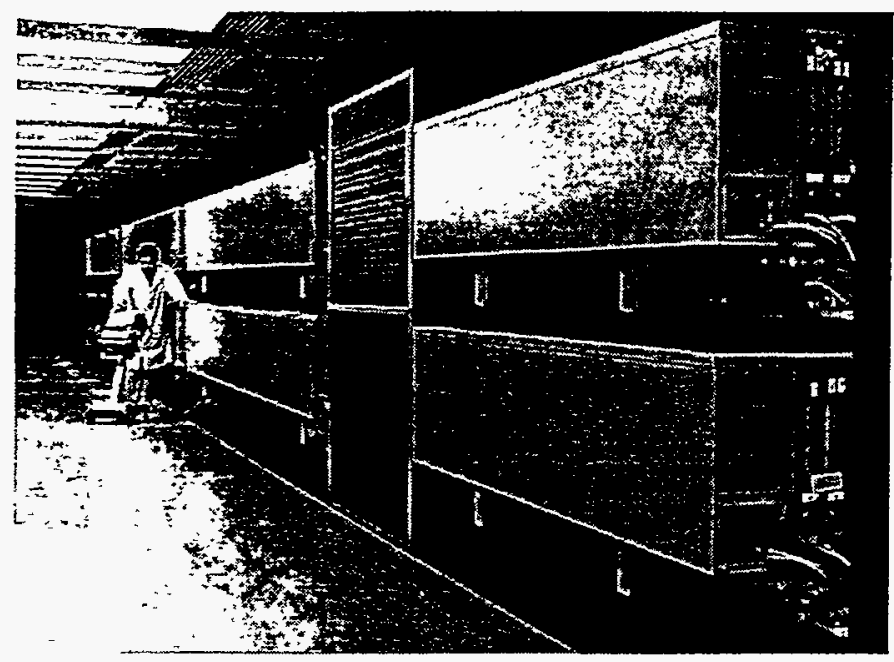

$>10 \mathrm{~kW}$ of laser light, and have been operated for millions of unit hours. Seen in the figure is the size and scale of two of the copper laser chains, each capable of $1.5 \mathrm{~kW}$ output power. The design is modular to allow efficient laser refurbishment.

In Fig. 4 is shown some of our dye lasers, which convert

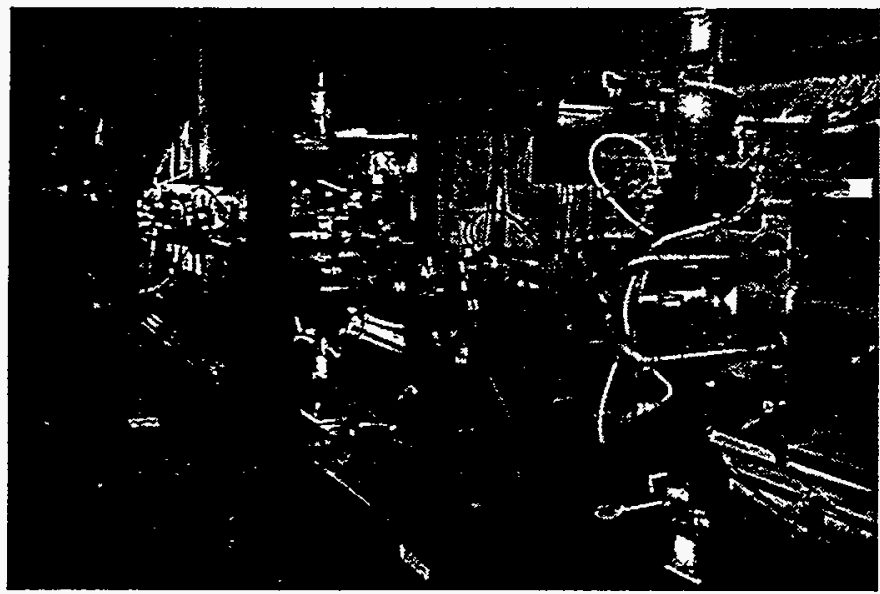

the copper laser light to a precise, narrow-bandwidth wavelength suitable for AVLIS. These systems have been used in round the clock operations in support of enrichment demonstrations, and to gather reliability data.

The separator systems, shown in Fig. 5, have been used to

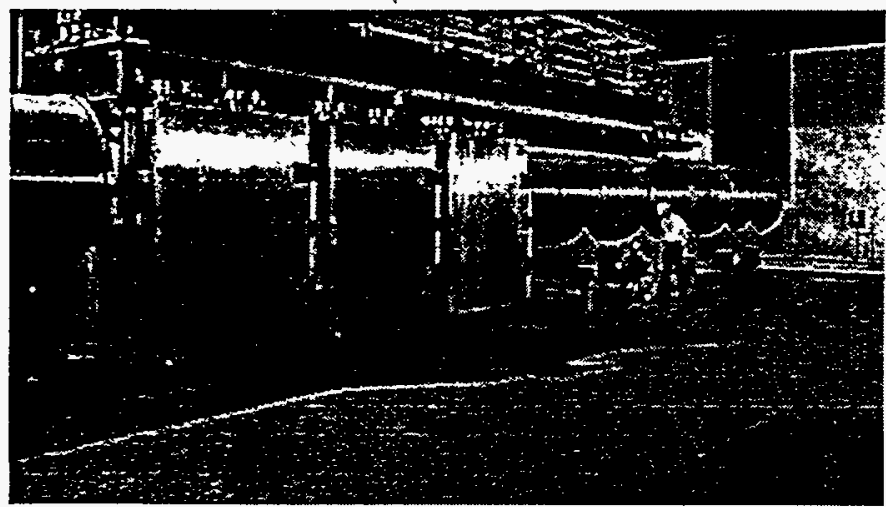

process metric tons of uranium. Integrated demonstrations have produced hundreds of kilograms of reactor grade uranium (enrichments between $2 \%$ and $5 \%{ }^{235} \mathrm{U}$ ). In addition, the facilities have been used for individual component development and also for the gathering of additional reliability data.

The program has also enriched multi-kilograms of several of the lanthanide series including erbium and gadolium as well as investigated enrichment schemes for a host of other elements.

\section{Present Status of the AVLIS Program}

By act of Congress a government corporation named the United States Enrichment Corporation (USEC) was formed and subsequently granted all rights to AVLIS technology previously owned by DOE. USEC has submitted a privatization plan to the President and Congress and expects approval this year. The USEC Board of Directors decided in 1994 to take steps toward the deployment of an AVLIS uranium plant. USEC is sponsoring at LLNL the completion of technology development and preliminary uranium plant design in FY96 and FY97.

Under USEC sponsorship, LLNL is also investigating other industrial and medical isotope separation programs for potential future business.

\section{Isotopic Purification of Lead}

Initial investigation into lead purification indicates technical and commercial feasibility by application of AVLIS technology developed for the uranium program. Spectroscopic studies have identified photoionization pathways which will efficiently and selectively ionize ${ }^{210} \mathrm{~Pb}$. Shown in Fig. 6 is the spectroscopic laboratory used for this work.

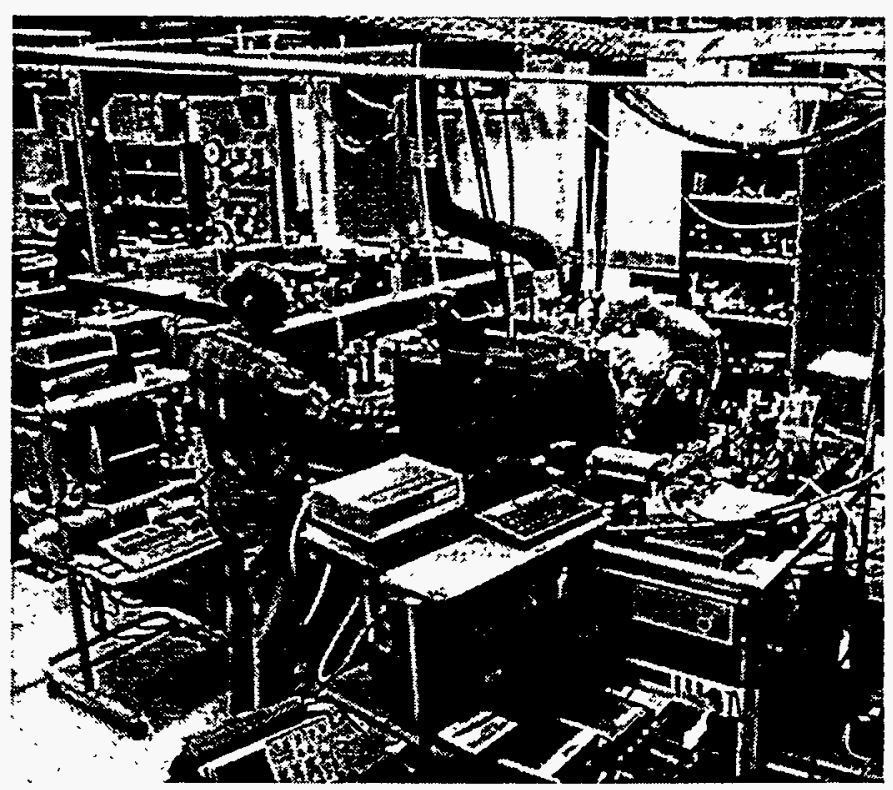

These studies also provide necessary data to specify the technical requirements of the laser system, and ultimately, the laser costs. For lead purification the required laser system is not available commercially. But by applying expertise gained in the uranium program, we are presently developing a multiple-color prototypical laser system that would be integrated into the purification system. The laser development 
facility is shown in Fig. 7. This facility will also be usable to

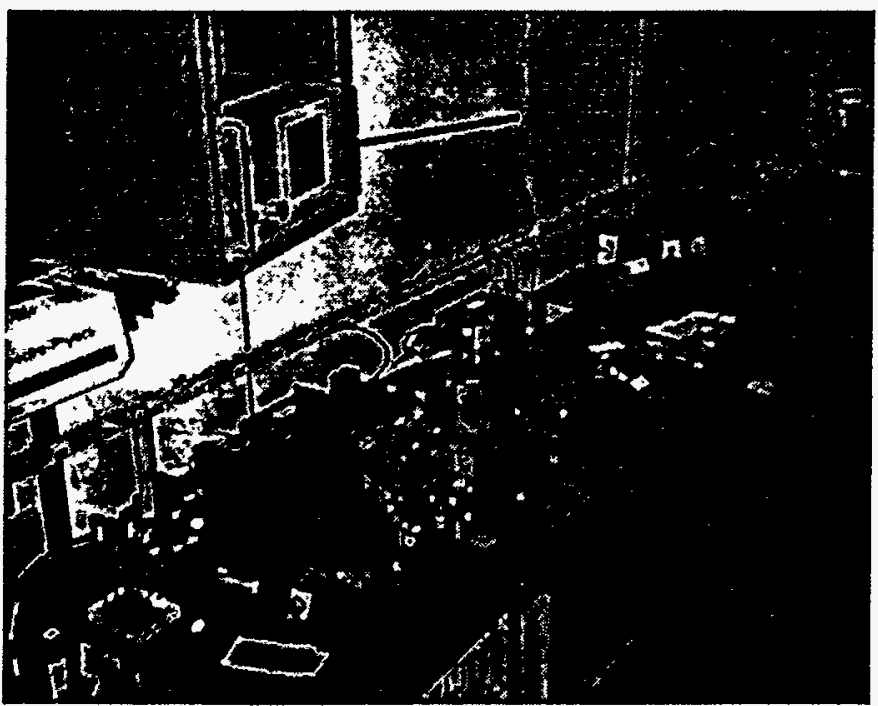

support a production system.

The market demand primarily determines the overall dimensions of the separator system, whereas some of the spectroscopic data - isotope shifts, hyperfine splittings, and the like - are required for a specific separator design. In Fig. 8 is shown the separator development facility that is presently being used to characterize the properties of the atomic lead vapor while varying source designs and design parameters. In

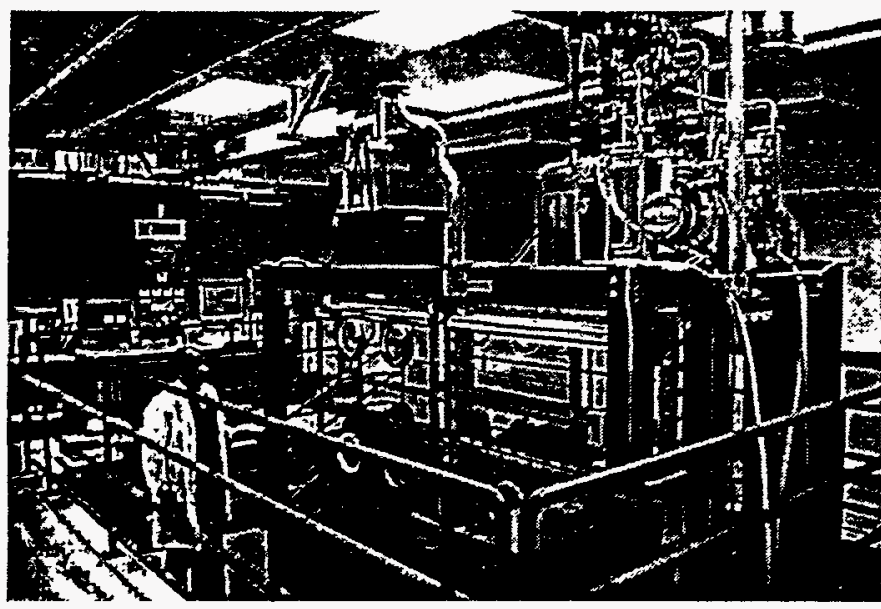

addition to the vapor property measurements, this facility also will be used to develop the Product and Tails collection hardware, and possibly for production at a later time.

This developmental phase is anticipated to extend through the current fiscal year where the prototypical laser and separator hardware required to process lead will be specified and developed. Presuming success and if sufficient commercial interest develops, we will carry out purification demonstrations during the first part of FY97 followed by the development of production-scale hardware later that year. In FY98, full scale production will begin. USEC will consider offering limited quantities of lead depleted in ${ }^{210} \mathrm{~Pb}$ in FY97, and ton quantities beginning in FY98.

Success is defined as much by the technical merits of the program as by the associated costs of the purification. The production costs will be well characterized as part of the development program. The major determining factors are the size of the laser system (i.e. the laser power requirements), the separator cost per unit throughput, and the labor costs required to run the facility.

\section{LLNL's $\alpha$-detection Capabilities}

The Isotope Sciences Division at LLNL routinely measures very low alpha activities of ${ }^{210} \mathrm{Po}$, a ${ }^{210} \mathrm{~Pb}$ granddaughter. A sample solution is spiked with a known amount of ${ }^{209}$ Po solution (which has been calibrated against a NIST-traceable standard). Spike and sample Po are chemically equilibrated and then spontaneously plated onto a silver planchet. Po activities are determined by alpha energy spectrometry using partially depleted silicon surface barrier detectors. Peak resolution for plated Po is $\sim 40 \mathrm{keV}$ FWHM, while the energy difference between ${ }^{209} \mathrm{Po}$ and ${ }^{210} \mathrm{Po}$ is greater than $400 \mathrm{keV}$. The use of a calibrated spike allows quantitative determination of ${ }^{210}$ Po activity without having to precisely characterize chemical yield, plating efficiency, plating thickness, and counting geometry. For 1-10 grams of industrial lead, the detection limit is better than 0.001 alphas/ hour $/ \mathrm{cm} 2$. The only disadvantage to the technique is that in order to relate ${ }^{210} \mathrm{~Pb}$ activity to measured ${ }^{210} \mathrm{Po}$ activity, the extent of secular equilibrium between parent and granddaughter must be known. For recently chemically processed samples, a second ${ }^{210}$ Po determination must be made several months after the first determination. For samples which have not been chemically processed for five half-lives of ${ }^{210}$ Po (at least two years), secular equilibrium can be assumed, and only a single measurement is required.

LLNL also has beta-counting facilities, and ${ }^{210} \mathrm{~Pb}$ can be determined by beta counting its ${ }^{210} \mathrm{Bi}$ daughter. Because ${ }^{210} \mathrm{Bi}$ has a much shorter half-life than ${ }^{210}$ Po ( 5 days vs. 138 days), secular equilibrium with ${ }^{210} \mathrm{~Pb}$ is established much more rapidly ( 1 month vs. 2 years). The ${ }^{210} \mathrm{Bi}$ technique involves adding stable $\mathrm{Bi}$ to the sample solution as a carrier and yield monitor, chemical separation of $\mathrm{Bi}$ from other beta and alpha emitters, and beta counting in a gas-flow proportional counter. Higher backgrounds, poorer energy resolution, and corrections for absorption and scattering make the ${ }^{210} \mathrm{Bi}$ technique less sensitive and less precise than the ${ }^{210} \mathrm{Po}$ technique for determining ${ }^{210} \mathrm{~Pb}$ activity. But the faster turnaround time is potentially more suitable for development and process applications.

\section{Conclusions}

Over the last two years we have built an R\&D facility eventually capable of production MT/y of isotopically purified lead. Much of the hardware is in place and the rest (both lasers and separators) is being developed. Furthermore, we have completed a spectroscopic investigation that has resulted in purification scheme that can in principle reduce the ${ }^{210} \mathrm{~Pb}$ content in lead to an industry-specified arbitrarily low value.

LLNL has alpha detection capabilities of immediate use to the semiconductor industry, and of potential use to characterize the lead that has been purified by the AVLIS process.

This work was performed under the auspices of the U.S. Department of Energy by Lawrence Livermore National Laboratory under Contract W-7405-ENG-48. 



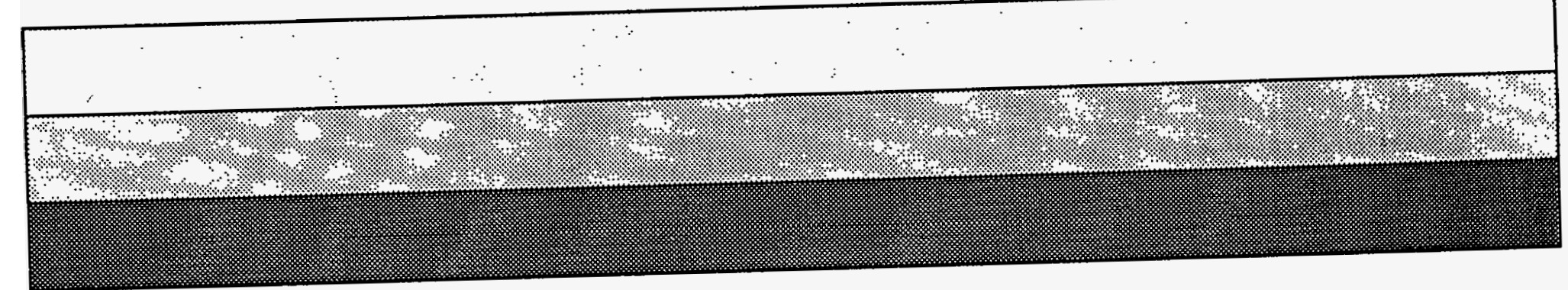

